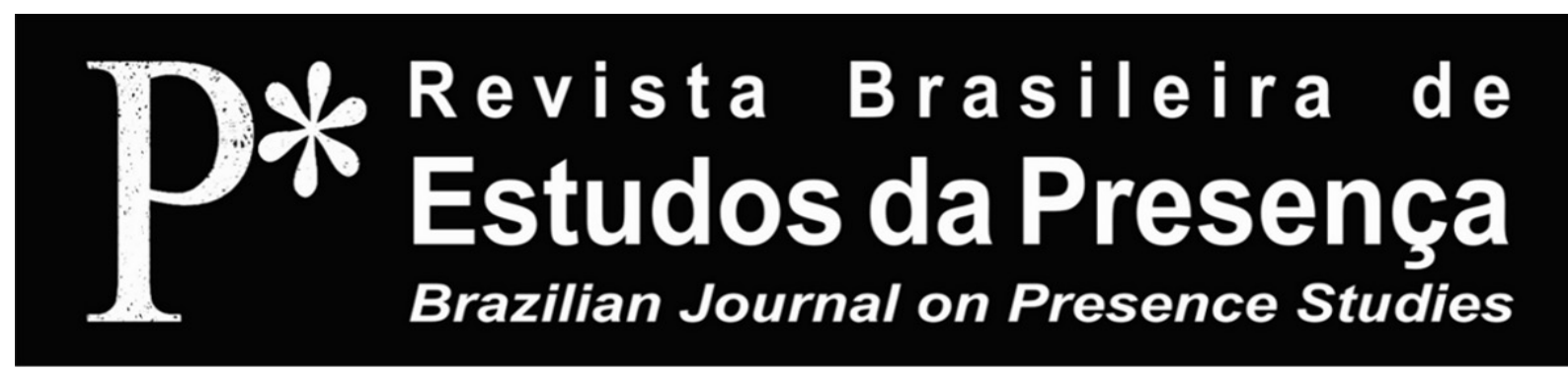

\title{
L'Hybride à l'Épreuve du Corps Dansant: étude de créations de la scène contemporaine anglo-saxonne
}

\author{
Federica Fratagnoli \\ Université de Nice Sophia Antipolis - Nice, France
}

RÉSUMÉ - L'Hybride à l'Épreuve du Corps Dansant: étude de créations de la scène contemporaine anglo-saxonne - Prenant comme point de départ l'expérience des artistes de la diaspora indienne en Angleterre, l'article propose de repenser le rôle et la signification du terme hybride dans le champ de la danse et de la création contemporaine. Appliqué sans distinction à toute sorte de création artistique à caractère multiculturel, ce terme véhicule une pluralité de sens parfois contradictoires. Le recours à des notions de biologie contemporaine fournira les outils théoriques nécessaires à une analyse des processus de création hybrides, permettant une lecture diversifiée d'expériences souvent perçues comme homogènes.

Mots-clés: Danse Contemporaine. South Asian Dance. Créations Hybrides. Diaspora Indienne. Morphogenèse.

ABSTRACT - The Hybrid and the Dancing Body: a study of the Anglo-Saxon contemporary stage - Taking as starting point the experience of the artists of the Indian diaspora in England, the article suggests rethinking the role and the meaning of the term bybrid in the field of dance and of contemporary creation. Applied without distinction to any kind of multicultural artistic creation, this term conveys a plurality of senses, sometimes contradictory. Notions of contemporary biology will supply the theoretical tools for an analysis of the hybrid processes of creation, allowing a diversified reading of experiences often perceived as homogeneous.

Keywords: Contemporary Dance. South Asian Dance. Hybrid Creations. Indian Diaspora. Morphogenese.

RESUMO - O Hibridismo e o Corpo Dançante: um estudo sobre criaçóes na cena contemporânea anglo-saxã - Ao tomar como ponto de partida a experiência dos artistas da diáspora indiana na Inglaterra, este artigo propóe repensar o papel e o significado do termo híbrido no campo da dança e na criaçáo contemporânea. Aplicado sem distinção a todo tipo de criaçáo artística de caráter multicultural, esse termo veicula uma pluralidade de sentidos, às vezes, contraditórios. $\mathrm{O}$ recurso a noçôes da biologia contemporânea fornecerá as ferramentas necessárias a uma análise dos processos híbridos de criação, permitindo uma leitura diversificada de experiências muitas vezes percebidas como homogêneas.

Palavras-chave: Dança Contemporânea. Dança do Sul da Ásia. Criaçóes Híbridas. Diáspora Indiana. Morfogênese. 
En se confrontant à la production discursive sur les nouvelles créations chorégraphiques indiennes, en Inde comme en Occident, on remarque que se répand l'utilisation de l'adjectif hybride appliqué sans distinction à tout travail artistique à caractère multiculturel. C'est au cours des années 1980 que la notion d'hybridité s'affirme dans le monde anglophone comme nouvelle manière historique et dynamique d'interroger les faits culturels. Assumant une dimension à la fois esthétique et philosophique, elle s'impose dans le champ de l'art comme une valeur positive et très prisée. Or, dans le domaine de la danse, cette notion apporte avec elle de multiples paradoxes et ambiguïtés. Véhiculant une pluralité de sens parfois contradictoires, le terme occulte la spécificité des processus créatifs et des esthétiques, souvent divergents, à l'œuvre sur les scènes contemporaines.

Prenant comme point de départ le contexte britannique - le nœud le plus fructueux de l'expérience artistique indienne en Europe - cet article se propose de repenser le rôle et la signification de ce terme ainsi que les incohérences qu'il soulève dans la pratique corporelle. Pour quelle(s) raison(s) le terme hybride est-il devenu le leitmotiv des créations de la diaspora indienne en Angleterre? Quelle(s) intention(s) et quelle(s) nécessité(s) ont motivé son usage? Et plus encore, le terme demeure-t-il pertinent pour un discours sur le corps et sur la danse? Il nous semble que la connotation donnée par la biologie du XIX ${ }^{\mathrm{e}}$ siècle, inscrite dans l'étymologie même du terme ${ }^{1}$, empêche la saisie de l'apparition des configurations sensibles qui animent la corporéité dansante et rend l'utilisation de ce terme inadéquate et problématique dans le champ de l'expérience corporelle. Seul le recours à des notions de biologie contemporaine qui, contrairement à la biologie du XIX ${ }^{\mathrm{e}}$ siècle, s'ouvre à une étude cognitive et phénoménologique de la vie humaine, fournit les outils théoriques nécessaires à ce type d'étude.

Suivant la pensée du philosophe Michel Bernard ${ }^{2}$, les pages de cet article envisageront le corps non pas comme une entité anatomique permanente et signifiante, mais comme un "[...] réseau sensorimoteur instable d'intensités, soumis aux fluctuations d'une double histoire symbolique: celle de la société ou de la culture à laquelle il appartient et celle de la singularité évènementielle et contingente de sa propre existence" (Bernard, 2001, p. 86). Le terme sensible, utilisé à plusieurs reprises au cours du texte, renverra d'emblée à l'expérience perceptive vécue par l'interprète 3 . Plus précisément, ce terme dési- 
gnera l'affinement sensorimoteur qui accompagne l'apprentissage d'un nouveau geste ou d'une nouvelle pratique de danse, permettant l'incorporation de coordinations et de qualités de mouvement jusqu'alors inconnues. Il nous semble qu'un changement d'optique du corps, pensé comme entité anatomique, à la corporéité, pensée comme entité sensible, permettra de lire les créations contemporaines de façon plus pertinente, mettant à jour une diversification d'expériences souvent perçues comme homogènes.

L'article s'organise en trois parties. Dans un premier temps, nous présenterons le contexte de la danse anglo-saxonne et nous analyserons l'apparition du mot hybride dans le domaine de la danse anglaise. Successivement, nous reviendrons sur l'apparition et la diffusion de la notion d'hybride dans les arts performatifs. Cela nous permettra de repenser la place de cette expression dans notre champ d'application, la danse, et de déceler les conséquences que la confluence de cultures physiques hétérogènes peut induire dans le domaine des pratiques corporelles. En dernier lieu, nous présenterons des études de cas spécifiques qui permettront de nommer certains des processus de créations hybrides présents sur les scènes contemporaines. Nous tenons à préciser que le paysage esquissé dans ces pages n'est pas représentatif de l'ensemble des créations propres à la scène contemporaine, assez diversifiée et marquée par des tensions parfois contradictoires. Toutefois, ce panorama pourra fournir des axes de réflexion utiles sur le sujet.

\section{Apparition du Mot Hybride dans le Contexte Anglo-saxon}

La Grande-Bretagne est sûrement le pays européen qui connaît la production de pièces chorégraphiques et de discours la plus remarquable au sujet de la diaspora indienne. Un mouvement institutionnalisé s'est imposé dans ce pays dès la fin des années 1960, rassemblant une communauté assez vaste d'artistes provenant des pays de l'Asie du Sud, autour d'un projet multiculturel très ambitieux. Nous avons expressément utilisé le terme Asie du Sud pour rendre compte de l'expression anglaise South Asian qui, dès les années 1980, désigne le panorama artistique indo-britannique. Précisons qu'en réalité les styles de danses indiennes en Angleterre sont désormais identifiés sous l'expression de South Asian Dance. Ce terme vise à souligner que les danses de l'Inde ne sont plus dansées seulement par 
les Indiens, mais par une myriade d'artistes, provenant de nations diverses, du Pakistan au Bangladesh, du Népal au Sri Lanka, de l'Inde à l'Afrique, sans compter ceux venant d'Europe. Problématisé par Avanthi Meduri dans un article paru en 2008 (Meduri, 2008), le terme South Asian témoigne du phénomène de transnationalisation et d'hybridation de la pratique, qui finit par superposer à l'identité locale de cette danse, propre à la nation indienne, une deuxième identité plus spécifiquement globale ${ }^{4}$. Avanthi Meduri démontre que cette transformation s'est concrétisée à travers la création d'un réseau institutionnel local-global, qui est aujourd'hui devenu une réalité très importante dans le contexte anglais. Plusieurs exemples peuvent être mentionnés à ce sujet. Citons les institutions d'Akademy, de Kadam ou de Sampad, qui visent à la propagation de la culture South Asian, avec une attention particulière destinée aux créations contemporaines. Ou encore Pulse $e^{5}$, journal de diffusion des actualités de la danse South Asian en Angleterre. Financée par le Arts Council of England, cette publication se propose explicitement de "[...] souligner que la Grande-Bretagne est un lieu d'événements pour la danse 'South Asian' et qu'elle impulse des tendances inter-

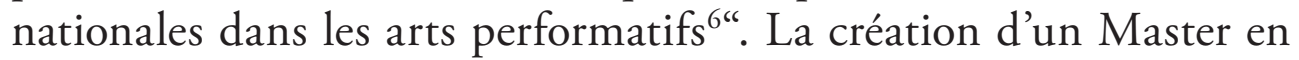
South Asian Dance Studies, au sein du département d'anthropologie de la danse de Roehampton University (London), s'accompagne de la mise en place de l'option pratique en South Asian Dance au sein de The Place, l'une des écoles londoniennes les plus renommées en danse contemporaine. Enfin, citons la création d'un certificat pour l'enseignement de la danse indienne, Imperial Society of Teachers of Dancing-ISTD correspondant au Diplôme d'Etat français pour les styles de danse Bharata Natyam et Kathak, et le programme estival qui se déroule à Leicester, Unlocking Creativity, laboratoire de créations artistiques contemporaines à partir des principes techniques des danses indiennes.

Ce que l'on vient de décrire est le fruit d'un long processus d'affirmation et d'enracinement progressif qui a été amorcé dès le début $\mathrm{du} \mathrm{XX}^{\mathrm{e}}$ siècle, en lien avec les premières migrations indiennes sur le sol anglais. Reçues et accueillies dans un premier moment sous le label exotique et ethnique, ces danses ont gagné de la reconnaissance et du prestige, jusqu'à être reconnues comme un courant à part entière, non plus subordonné mais parallèle au courant principal de la danse contemporaine britannique (Khan; Sundaram; Wollaston; Ray, 2001). 
Or, le terme hybride fait son apparition dans le domaine de la danse anglaise vers la fin des années quatre-vingt. Shobana Jeyasing, une danseuse née à Chennai (Inde) en 1957 et installée en Angleterre à partir de 1981, est à l'origine de cet emprunt linguistique. Formée à la danse indienne de style Bharata Natyam, elle suit un master en littérature anglaise - MA in Shakespeare Studies à l'Université de Sussex - qui l'introduit aux auteurs postcoloniaux, en premier lieu Homi Bhabha et Stuart Hall, dont elle se déclare à plusieurs reprises redevable. Shobana Jayasing se produit initialement dans des solos de danse traditionnelle, sans toutefois connaître un grand succès public. Vers la deuxième moitié des années quatre-vingt, l'intérêt pour les auteurs de la diaspora indienne et pour les théories postcoloniales l'incite à reporter son attention sur les possibilités expressives du Bharata Natyam dans le contexte occidental (Meduri, 2008, p. 305-307). Elle se présente alors sur la scène artistique londonienne avec un projet esthétique très ambitieux: rendre compte du déplacement identitaire enduré par les pratiques corporelles indiennes dans l'époque postcoloniale 7 . Afin de concrétiser ce projet, elle fonde en 1988 la Shobana Jeyasing Dance Company, destinée à devenir l'une des compagnies les plus connues et appréciées dans les milieux de création chorégraphique indienne de l'Angleterre.

Cependant, dans ses toutes premières créations, comme Configurations (1988), Correspondences (1990) ou Making of Maps (1992), le concept d'hybridité a plus à voir avec un idéal théorique qu'avec une véritable expression corporelle. Au début de la recherche corporelle de Shobana Jeyasing, la mise en œuvre des interactions avec la culture occidentale se limitent à une simple décontextualisation du Bharata Natyam, circonstance qui incite Sanjoi Roy à parler "[...] d'urbanisation de la pratique" (Roy, 2003, n.p.). Citons comme exemple manifeste la pièce qui l'a rendue fameuse, Duets with Automobiles, conçue pour la vidéo. Cette pièce, qui date de 1993, se limite à une réorganisation du langage traditionnel du Bharata Natyam dans un espace urbain. Les enchaînements propres à ce style de danse sont déplacés à l'intérieur d'un bâtiment typiquement londonien, inscrivant les mouvements des danseuses dans un contexte étranger (Lopez y Rojo, 2006). Cet exemple assez extrême permet de soulever un problème technique, qui intéresse la totalité des créations dites hybrides. Shobana Jeyasing, et la plupart des chorégraphes des années quatre-vingt, construisent des projets dits bybrides en 
travaillant avec des danseurs qui possèdent exclusivement une pratique de danse indienne. C'est seulement autour de la moitié des années 1990 que Shobana Jeyasing commencera à collaborer avec des danseurs et des danseuses qui ont reçu une double formation: ils maîtrisent une technique de danse contemporaine et connaissent l'un des styles de danse et/ou d'art martial du subcontinent indien. Or, c'est exactement cette formation multiple - que sous-tend une pratique sensible et perceptive diversifiée - qui permettra le jaillissement d'interférences kinesthésiques qui déplacent le langage indien vers d'autres domaines. Nous reviendrons sur cette question dans la dernière partie de l'article. À partir de cette deuxième période - la seconde moitié des années 1990 - les caractères du projet corporel postcolonial de Shobana Jeyasing se clarifieront et assumeront une cohérence avec les principes théoriques qu'elle soutient. Dès lors, le label hybrid(ity) sera employé pour la quasi-totalité des travaux d'expérimentation qui cherchent à négocier l'identité corporelle, en dehors du homeland originaire.

Comme nous le rappelle Stéphanie Jordan (2001, p. 114), c'est pendant la conférence Re-inventing Britain tenue à Londres en 1997, que le terme hybrid(ity) a été envisagé comme l'expression la plus appropriée pour parler des investigations interculturelles dans le champ chorégraphique. Ce terme, emprunté à la réflexion de Homi $\mathrm{Bhabha}^{8}$, permettait, selon les participants, de maintenir une certaine ouverture ainsi qu'une certaine complexité de signification par rapport au terme précédemment employé dans ce domaine: fusion. Évoquant la réunion d'éléments distincts en un tout homogène, $f u$ sion renvoyait à l'idée d'un processus qui donne vie à un objet dont la structure interne était unitaire et statique. L'expression hybrid(ity), en revanche, est porteuse de l'idée de mobilité et de réorganisation permanente, qui l'associe à une dynamique en devenir, où les éléments se maintiennent tout en se mélangeant entre eux. Dans cette deuxième acception, l'idée de bornes et de confins perd justement sa pertinence. Ce n'est pas la forme qui est en jeu mais la dynamique.

Utile dans un moment de renégociation identitaire et de transition postcoloniale, il nous semble que l'utilisation du terme hybride demande aujourd'hui à être questionnée. Les différents types de langages corporels et de propositions chorégraphiques enfermés dans les mailles de son label rendent son utilisation vague et parfois inopportune. 


\section{Le Terme Hybride}

Depuis une vingtaine d'années, nous assistons à un phénomène de "[...] prolifération des hybrides", pour reprendre une expression de Bruno Latour (1997, p. 7). Le terme hybride est devenu un terme courant et ordinaire, employé dans des contextes aussi différents que l'économie, les nouvelles technologies, la cuisine, la littérature, les arts plastiques ou performatifs, pour n'en citer que quelques-uns. Dans chacun de ces contextes, ce terme assume des acceptions souvent divergentes, lui conférant ainsi des identités diverses.

La rhétorique de l'hybride s'affirme de façon conséquente au cours des années quatre-vingt comme une nouvelle manière historique et dynamique d'interroger les faits culturels. Strictement associé à l'émergence du discours postcolonial et à sa critique de l'impérialisme culturel, ce concept canalise les réflexions et les tensions qui animent le travail intellectuel des Subaltern Studies et des Cultural Studies. Après avoir fait partie de champs plus proprement sociologiques et historiographiques, la notion d'hybride s'insinue dans le domaine de la littérature. Les connexions entre texte et pouvoir, qui remettent en cause la façon d'articuler la présence de l'autre dans l'histoire culturelle, légitiment ce déplacement. Tout texte est en fait pensé comme source de pouvoir et toute textualité comme lieu privilégié de représentation et de résistance. La notion d'hybride - à côté du principe du multiculturalisme - se précise comme l'une des notions clefs des réflexions de ces intellectuels, résumant la nécessité d'analyser la superposition des cultures et des connaissances propres à l'époque postcoloniale. Une fois décontextualisé au sein du domaine littéraire, le terme hybride ne tardera pas à s'imposer dans le champ artistique, où il témoignera de la rencontre et de l'interaction de pratiques artistiques provenant de contextes divers.

Or, dans le domaine des arts performatifs le terme hybride fait son apparition pour nommer l'implication des nouvelles technologies, en particulier du numérique, visant à imposer un nouvel ordre visuel, qui simule le réel plutôt que de le définir. On retrouve donc ce terme dans la vidéo-danse, ou danse à l'écran, et dans toute performance d'art numérique ou installation d'art contemporain'. Dans le domaine de la danse contemporaine, le terme a été généralement employé pour rendre compte de créations chorégraphiques qui ont recours à d'autres arts ou technologies. Récemment, François Frimat 
(Frimat, 2010) pousse la réflexion un peu plus loin, employant le terme hybride pour rendre compte de tous les éléments qui, sur les scènes contemporaines, perturbent l'identité de la représentation et déroutent la perception du spectateur.

Face à cette prolifération des hybrides, il nous semble essentiel de penser et analyser l'hybridation, non pas comme un processus général, mais comme un processus "[...] impliquant au contraire une multiplicité de processus spécifiques" (Molinet, 2006, p. 3). "L'hybridation n'étant pas un objet en soi, mais l'action de transformer et modifier à la fois les objets, les outils, les pratiques, agissant sur les formes de construction ou réception du réel” (Molinet, 2006, p. 2-3) explique Emmanuel Molinet. C'est donc seulement en examinant les processus qui sous-tendent l'acte de création qu'il deviendrait possible d'approcher la complexité de ce phénomène et de rendre compte de la spécificité de chaque forme hybride.

\section{Comment un Corps se rend Sensible à une Culture Autre}

Repenser le concept d'hybridation est requis par la corporéité et par le regard sensible que nous avons décidé d'y porter. En tant que réseau de connexions sensibles et de textures énergétiques, le corps nous oblige à nous confronter à des questions spécifiques, qui sont de l'ordre de l'incorporation, de l'élaboration des flux et de la perte des limites. La rencontre entre deux expériences corporelles ne peut donc pas être envisagée comme un moyen de résoudre une dualité foncière. Elle est plutôt un moyen de repenser comment la corporéité d'un danseur se rend disponible pour incorporer (embodiment) des coordinations, des états toniques, des sensations qui appartiennent à une pratique culturelle autre et jusqu'alors inconnus.

Or, deux points nous semblent dignes d'être explicités. En premier lieu, nous n'adhèrerons pas expressément à une pensée de l'hybride qui se donne comme volonté de rapprocher et réconcilier les contrastes. Il nous semble en fait que l'expérience corporelle du sujet trouve son expression la plus convaincante dans les cas où elle jaillit de façon inattendue et involontaire. Loin de vouloir l'envisager comme un geste prémédité, résolutif, elle se présentera plutôt comme une conséquence, un effet inattendu et pourtant ostensible. La notion de corporéité impose en fait de reconsidérer la préméditation propre à tout phénomène d'hybridation pensée d'un point de 
vue culturel. Serait-il possible que la corporéité se donne comme lieu d'une genèse qui échappe à toute volonté? Quels enjeux expressifs pourraient induire une telle a-programmaticité corporelle? Souvent le sujet qui danse décide de façon imprévue et irréfléchie, laissant apparaître de manière anodine et presque négligeable les effets de la rencontre. Dans ces cas, une véritable génération spontanée surgit sans préavis. En deuxième lieu, cette approche empêchera de penser l'hybride comme la genèse d'un objet cohérent et harmonieux, qui dénouerait les contradictions irrésolues dans tout acte de transmission et d'incorporation du geste dansé. L'hybride aurait alors plutôt tendance à laisser émerger l'incohérence, le déséquilibre, la rupture, bref à faire vaciller toutes les certitudes pourtant déjà mises à l'écart par les corporéités afin de permettre l'accueil de l'autre. Il s'agirait alors d'une "[...] expérience de la désappropriation, de l'absence et de l'incertitude" (Laplantine; Nouss, 2001, p. 10) qui remet en question l'individu et la perception de sa corporéité. Par conséquent, l'hybride serait, avant tout, porteur d'un processus de dépouillement et de renoncement. La rencontre avec le divers demanderait un éloignement de ce que l'on est, une dépossession qui seule permettrait éventuellement l'accès au territoire inconnu et étranger de l'altérité. La tension mise en acte par le mouvement d'aller vers fragilisera et rendra instable le sujet de départ, qui se découvrira incomplet et fissuré. Dans la matière sensible et perceptive du corps, l'autre ne peut s'insinuer quau travers d'un mécanisme de corrosion des automatismes gestuels, créant des interférences et des lieux de syncope. L'instabilité et le réajustement continus empêchent le mécanisme de se scléroser, permettant ainsi d'échapper à la répétition $d u$ même et à la persistance d'habitus. Dans ce sens, l'expérience hybride coïnciderait davantage avec un état d'incertitude, d'égarement et enfin, éventuellement, de reconquête de soi. Il s'agirait d'un processus qui nie et réfute tout ce qui fixe, finalise, résout, révélant les figures qui jaillissent de cette réorganisation provisoire directement confrontée à l'informe. Reprenant les mots de Suely Rolnik, la rencontre avec l'autre instaurerait finalement le "[...] démontage de notre contour, de notre image corporelle, pour nous aventurer dans le processus bouillonnant de notre corps vibratile, sans image" (Rolnik, 2004, p. 4). Condition nécessaire et indispensable à toute complexification perceptive, l'état de vulnérabilité corporelle permettrait ce "[...] voyage vers cet au-delà de la représentation" (Rolnik, 2004, p. 4). 
La lecture sensible proposée dans ces pages prendra appui sur la théorie de la morphogenèse élaborée par Humberto Maturana et Francisco Varela. Leurs réflexions permettront d'envisager la rencontre de sensibilités hétérogènes non seulement comme contradiction jamais résolue ni aboutie, mais aussi comme système de réorganisation complexe qui réoriente les éléments à partir d'une forme conçue comme résonance.

\section{L'Émergence d'une Forme comme Processus Relationnel: la théorie de Humberto Maturana et Francisco Varela}

Le corps se construit par inscription culturelle. Un apprentissage constant et souvent inconscient détermine l'existence de cet organisme. Comme tous les systèmes complexes présents dans la nature, le corps réélabore les interférences provenant de l'environnement extérieur, intégrant ces éléments et donnant ainsi vie à des nouvelles configurations. Le concept d'autopoiesis, élaboré par les biologistes chiliens Humberto Maturana et Francisco Varela, relève de cette qualité auto-organisatrice propre aux systèmes complexes ${ }^{10}$. Provenant

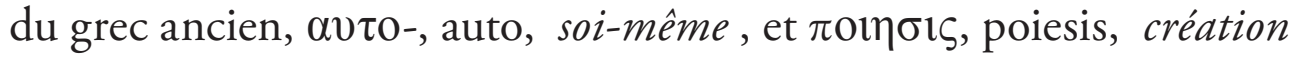
ou production, ce terme désigne un être qui s'auto-organise et qui arrive à gérer les obstacles qui minent son fonctionnement. À partir des années 1970, ce néologisme a été utilisé par Maturana et Varela afin de nommer la condition d'existence des êtres vivants pris dans une production continue d'eux-mêmes. D'après cette théorie, le caractère fondamental des systèmes vivants est de posséder une structure organisée. Un fonctionnement complexe et raffiné permet de garder et de régénérer dans le temps l'autonomie propre au système et à sa survivance. Pour cette raison, toute variation de l'environnement est assimilée dans le plus court délai possible afin de ramener l'organisme à l'unité. Cela se fait à travers des dispositifs qui contribuent à la régénération et au maintien de l'être vivant. L'autopoiesis serait donc la possibilité propre à tout système de réélaborer son identité grâce à des processus intérieurs qui auto-reproduisent et transforment leurs propres composants.

En tant que système complexe, le corps est un organisme qui s'ordonne et réagit selon les principes de l'autopoiesis. Cela signifie que les interférences ou les bruits qui s'insinuent dans sa structure interne déclenchent à chaque fois un dispositif de réorientation qui 
les incorpore à un niveau supérieur. Ce cadre théorique permettrait donc de penser l'incorporation d'un nouveau geste ou d'une nouvelle pratique corporelle, par le danseur-interprète, comme une forme de bruit, une perturbation interne dans la façon de sentir. Ce trouble mettrait en acte un réel bouleversement du cadre perceptif, contraint de se réorganiser pour négocier une nouvelle intégrité à un niveau supérieur. Bien évidemment, cette modification sensible sera perceptible dans de légers déplacements, des perspectives inédites, des circuits impalpables, qui donneront vie à un nouvel agencement postural et moteur.

Notre sujet d'études exige de nous intéresser aux répercussions physionomiques et visibles de cette réorganisation intime, qui se fait au moment de l'incorporation d'un nouveau geste ou d'une nouvelle coordination. Dans le cas des créations hybrides, à quoi le regard du public se trouve-t-il confronté? Quelle physionomie assume cette réorganisation sensible d'univers corporels? Est-il possible de parler encore de forme? C'est pour répondre à ces questions que nous ferons appel, au fil de ces pages, au concept de morphogenèse. Dans le champ de la biologie, ce terme indique l'émergence des formes qui caractérisent un organisme vivant, soit du point de vue évolutif, soit du point de vue du développement ontogénétique. D’après les mots de Varela (2001), la plus grande découverte de la science des dernières années a été justement de comprendre comment penser l'émergence d'un phénomène, pour expliquer le passage d'un niveau local à un niveau global. L'idée que tout ce qui existe soit relié à une existence matérielle et soit donc saisissable en termes objectifs est un modus pensandi propre à la société occidentale. Cette posture a caractérisé les acquis de la philosophy of mind américaine qui s'est principalement consacrée à donner des définitions de catégories et d'objets. Cependant, cette attitude consistant à pointer du doigt et matérialiser dans un objet concret et identifiable tout phénomène, pose désormais des soucis. Les spécialistes des sciences exactes se trouvent davantage confrontés à des réalités nouvelles, qui ne peuvent pas être nommées ni saisies de façon concrète. Pour cette raison, les dernières recherches en la matière tendent en fait à revoir la position analytique, qui laisse inexpliquée l'émergence de plusieurs phénomènes. Pour expliquer ces nouvelles réalités, Varela recourt à un exemple (Varela, 2001) banal mais qui éclaire de façon nette la question: 
Dans l'atmosphère circulent d'innombrables particules d'air et d'eau qui soudain par un phénomène d'auto organisation - ça c'est le mot clef - deviennent une tornade, un objet qui apparemment n'a pas une vraie existence, puisqu'il existe seulement dans les relations de ses composantes moléculaires. Cependant son existence est confirmée par le fait qu'il détruit tout ce qu'il trouve sur son chemin. Donc il est un objet curieux (Varela, 2001, n.p.).

Pour saisir la portée de cet exemple, il faudrait avant tout se demander qu'est-ce qu'une tornade? L'utilisation de ce mot n'exprime rien, sinon la complexité d'un phénomène qui est abstrait et insaisissable. La tornade possède, bien évidemment, une identité et une unité, mais où se situe-t-elle? Il est possible d'appréhender les particules d'air et d'eau, singulièrement, une par une. Mais il sera également très difficile de pouvoir indiquer leur mode d'être ensemble. Comme le remarque justement Varela, le passage de la partie au tout introduit une catégorie ontologique, une façon d'être. La tornade est un élément qui est émergent; impossible de le saisir et d'affirmer qu'il existe en soi. Il s'agit plutôt d'un état, d'une manière d'apparaître au monde. Rien d'autre. Cette évidence pousse Varela et beaucoup d'autres scientifiques contemporains à reconnaître la présence de nouvelles réalités que la science n'est pas encore arrivée à nommer et à appréhender.

Évidemment ces questionnements résonnent de manière très actuelle dans certains spectacles de danse contemporaine qui cherchent à faire interagir deux ou plusieurs pratiques corporelles. Ces créations posent le spectateur devant l'impossibilité de cerner la complexité de la corporéité du danseur. Issues d'un état et d'un travail sensible et proprioceptif plutôt que d'une forme, ces corporéités mettent en jeu les parties les plus subtiles et les moins visibles de l'identité corporelle. Comment, alors, percevoir la complexité d'un mouvement qui résulte de l'interaction de plusieurs pratiques corporelles? Comment localiser l'identité de ce mouvement, dans la mesure où "[...] son identité n'a pas un locus, mais une collocation spatio-temporelle" (Varela, 2001, n.p.)? Quel nouveau type d'identité devrait-on reconnaître à ce corps et à ces gestes ? C'est en effet la question de l'identité qui est en jeu dans l'émergence d'un phénomène. Reprenant les mots de Varela, les processus d'auto-organisation qu'il met en lumière "[...] donnent vie à un niveau auquel il faut reconnaître une identité spécifique" (Varela, 2001, n.p.). Dépourvue d'existence substantielle et 
matérielle, cette identité se présente sous la forme d'un processus strictement relationnel. Reprenant l'exemple de la tornade, nous pouvons affirmer qu'elle n'existe qu'à travers les relations de ses composantes moléculaires. Cette constatation conduit Varela à penser qu'il faudrait alors "[...] redéfinir la notion d'identité en termes d'interaction plutôt que d'extension dans une forme" (Varela, 2008, n.p.) et plus intéressant encore, qu'il faudrait "[...] déceler quels sont les types de mécanismes de résonance interne qui vont faire émerger une forme plutôt qu'une autre" (Varela, 2008, n.p.).

L'idée de forme envisagée par le biologiste sud-américain s'éloigne donc des paramètres figuratifs, visuels et graphiques à partir desquels nous avons le réflexe $\mathrm{d}$ 'interpréter les phénomènes. L'émergence d'une forme serait plutôt dépendante d'un mécanisme de résonance interne, qui prend vie à partir de l'interaction des substances qui composent la matière. Son observation fine et sensible, qui s'intéresse avant tout à l'état de la matière, le conduit à envisager un nouveau type de forme. Désignée dans les termes de résonance, cette configuration virtuelle serait à la base de l'identité de toute substance et de sa figuration extérieure. "La forme [...] au sens de cohérence donnée par cette espèce de résonance interne, est comme dans le modèle du style 'système immunitaire' où il n'y a pas de forme au sens physique, littéral, mais au sens d'une identité de résonance" (Batt; Abrioux; Cordesse, 1990, p. 175). D’après cette interprétation, il s'avèrerait possible de distinguer deux sortes de formes: d'un côté, la forme au sens où nous l'entendons habituellement. Elle correspondrait à une figure, à une image bien reconnaissable et repérable, qui trouve encore ses références directes dans le domaine physique et matériel; de l'autre, une forme virtuelle, qui échappe à l'évidence d'une identification physionomique. Ce type de forme est rendue possible par une identité de résonance et par des relations-interactions pratiquées au sein d'un acte d'auto-organisation. Évidemment le concept de résonance déstabilise la notion classique de forme - et toutes les notions apparentées - qui d'emblée apparaissent restrictives et aléatoires.

La majorité des créations que la scène contemporaine définit comme hybrides restent liées à un concept de forme classiquement entendue, ne parvenant que dans de rares cas à fragiliser la posture corporelle des danseurs. C'est l'attachement aux signes propres à la culture de l'Inde qui instaure une dépendance presque obsession- 
nelle à l'idée de forme classiquement entendue, empêchant donc l'affirmation d'une rencontre qui touche l'expérience kinesthésique et sensori-motrice de l'interprète. Le cadre théorique que nous avons dessiné constitue un outil de réflexion utile au domaine de la danse indienne. Il nous permettra de reconsidérer l'importance accordée au vocabulaire, et d'opérer une distinction entre les créations qui émergent comme formes de résonance des créations plus conventionnelles qui restent prises par une idée de forme figurative. Le chapitre suivant présentera quelques exemples choisis, tirés de la scène contemporaine anglaise. Ces exemples permettront d'élucider et clarifier nos affirmations.

\section{Créations Hybrides sur les Scènes Contemporaines: déterrito- rialisation, fragmentation, résonance}

La plupart des créations hybrides des artistes de la diaspora indienne restent tributaires d'un même souci. Elles continuent à se confronter à un vocabulaire d'origine très prégnant, qui impose un modèle de reconnaissance fixe. Un grand nombre de ces créations reste attaché à une idée de forme figurative, classiquement entendue, se confrontant avec difficulté à la force des signes de la tradition. Tout en voulant expérimenter de nouvelles voies qui se mesurent au contexte contemporain, ces pièces appréhendent une perte identitaire.

À titre d'exemple, nous pourrions citer le spectacle de Nina Rajarani, Quick!, qui présente un dispositif de création similaire aux premières pièces de Shobana Jeyasing. Ce spectacle a gagné en 2006 The Place Prize, une importante compétition chorégraphique subventionnée par Bloomberg. Il s'agit d'une pièce de quinze minutes, interprétée par huit danseurs de la compagnie, tous dotés d'une solide formation en Bharata Natyam, style de danse classique du sud-est de l'Inde. La chorégraphie est basée sur la représentation du milieu acharné et compétitif des hommes d'affaires londoniens. Les danseurs, habillés comme des businessmen - chemise blanche, pantalons gris et cravate - mettent en scène la vie intense et mouvementée de la métropole anglaise. Accompagnée par une musique karnatique live, la chorégraphie s'organise à partir de mouvements très rapides, exécutés sur un tempo très soutenu. Tous ces mouvements sont tirés du vocabulaire du Bharata Natyam traditionnel. Aucune déviation de la forme n'est permise aux danseurs, qui intègrent et représen- 
tent à la perfection le système classique. D'ailleurs, la compagnie de Rajarani - Srishti - se présente comme une compagnie qui “[...] garde l'authenticité de la danse et de la musique indiennes classiques pendant qu'elle présente ces formes de façon innovante dans un contexte contemporain ${ }^{11 “}$. Le seul élément qui crée un décalage par rapport à un spectacle classique, témoignant du dialogue avec le contexte londonien et d'une certaine innovation perceptive, concerne les costumes et la scénographie. Les corporéités et les postures des danseurs restent formellement inscrites dans la démarche classique, sans en être perturbées. Le processus hybride se limite ici à la création d'un paysage insolite, à une décontextualisation, qui ne déstabilise nullement le style et la forme de cette danse et, par conséquent, le système perceptif des danseurs.

Or, d'autres créations de la scène contemporaine cherchent à franchir les bornes de cette construction identitaire, bouleversant les repères propres à une représentation du mouvement iconique et figurative. Ces pièces se présentent comme un assemblage de signes et de citations gestuelles - aisément reconnaissables car issues de la forme originaire, même si elles sont brisées, détachées, déplacées, déformées et recomposées.

Proposons comme exemple la pièce Flicker, crée par Shoban Jeyasing en 2005. Cette pièce a l'ambition de faire dialoguer et interagir le Bharata Natyam et la danse contemporaine. Or, la quasitotalité des enchaînements chorégraphiques qui composent cette pièce sont construits à partir de séquences de mouvements traditionnels (adavus) fragmentées et réorganisées à partir d'une logique contradictoire. Plusieurs passages de cette pièce permettent d'apercevoir la position de base des jambes du Bharata Natyam (aramandi - demi plié) et des bras (nartiarambe), ainsi que le mouvement propre au cou, qui oscille alternativement de droite à gauche (adami), les frappes de pieds, ou encore certains adavus dans leur intégrité - dont nous pourrions citer, à titre d'exemple, le mouvement utilisé pour clore une section de danse abstraite (tirmanam), connue sous la vocalisation ta-dim-ghi-na-tom ou un enchaînement de mouvements sautés connu comme tat-tei-tam, dit-tei-tam. Cependant, ces images issues de la tradition indienne sont généralement utilisées en introduisant des variations ou des variantes: cassés, fragmentés ou rendus asymétriques, ces gestes s'inscrivent alors de façon insolite dans la corporéité 
des danseurs, se superposant à des mouvements plus proprement contemporains. Sans jamais parvenir à créer une réelle intégration sensible, la corporéité laisse émerger et cohabiter à un même niveau les deux signatures originaires des danses incorporées. Les limites et les marges entre ces deux expériences corporelles et identitaires restent bien repérables. Cette façon de faire interagir Bharata Natyam et danse contemporaine reste probablement la plus répandue dans le panorama contemporain.

D'autres artistes de la scène contemporaine poussent leur recherche plus loin. Leur intérêt porte sur les interactions qui engagent de façon plus intime la corporéité dansante et qui impliquent donc une remise en discussion du système perceptif propre au corps.

Un exemple significatif de ce type d'interaction est représenté par les créations du danseur et chorégraphe anglo-bangladais Akram Khan. Dans ses premières pièces, il laisse interagir de façon sensible ses deux expériences kinesthésiques - le khatak et la danse contemporaine - donnant vie à un langage spécifique, qui prendra le nom de contemporary kathak. Ce langage, "[...] basé sur (l)a découverte d'éléments 'des/ordonnés' régis par la confusion" (Piccirillo, 2008, p. 30), constitue encore aujourd'hui la marque constitutive de ses créations.

Une pièce comme Fix, un solo de 1999, illustre très clairement ce processus d'interaction. Cette pièce sonde les dynamiques et les mouvements circulaires qui caractérisent la danse kathak, en les faisant résonner dans une perspective contemporaine. Tout en restant fidèle aux principes dynamiques dictés par le kathak - d'une part, l'accumulation d'énergie produite au cours de mouvements répétitifs et en crescendo; d'autre part, le mouvement de dispersion, qui propage l'énergie vers les extrémités du corps, voire la périphérie de l'espace - le geste d'Akram Khan fuit les références rassurantes de la forme pour s'aventurer à son insu dans un territoire et une texture hétérogènes. Il est à remarquer qu'à la différence d'autres chorégraphes, Akram Khan bénéficie d'une double formation. La danse contemporaine ${ }^{12}$ s'est superposée à sa formation en $\mathrm{kathak}^{13}$, induisant une refondation des frontières du sensible dans son propre corps. Ces deux inscriptions dynamiques et stylistiques ont contribué à rendre complexe et parfois ambigu son statut de danseur, placé entre deux communautés et donc entre deux formes d'expression identitaire. 
Il est important de souligner que Fix, au même titre que les pièces de sa première période, se pose comme une réflexion spontanée et involontaire sur les principes structurels du kathak et de la danse contemporaine. Akram Khan affirme à plusieurs reprises qu'il ne travaille pas directement sur son propre corps, mais qu'il le laisse travailler seul: "J'avais réalisé que mon corps était en train de dicter ses propres décisions, de façon inconsciente, et qu'un langage en émergeait, comme une balance entre deux mondes ${ }^{14 “}$ (2003, n.p.). Son langage chorégraphique est le fruit d'un processus involontaire, qui n'est pas étudié et surtout qu'il ne revendique pas en tant que tel. Loin de vouloir rapprocher et réconcilier deux pratiques distinctes, Akram Khan assiste plutôt à la réorganisation sensible entamée par sa corporéité. Pour cette raison, il refuse le terme fusion au profit de celui de con-fusion (Mohaiemen, 2003), plus approprié à nommer l'origine de son langage performatif.

Il faut aussi remarquer que dans son processus de création un privilège particulier est accordé aux sensations. Cette démarche donne vie à un langage chorégraphique affranchi d'expédients visuels stéréotypés, qui évincent la citation classique et ponctuelle des codes. C'est à cet égard que la question de la reconnaissance des danses de l'Inde - et donc du regard porté sur elles - acquiert une importance primordiale. Si, d'un côté, la stratégie employée par Akram Khan a permis de réorganiser un espace corporel inédit pour le kathak en lui accordant une autonomie auparavant impensable, de l'autre, il a évidemment déstabilisé la réception de ce style. La recherche d'Akram Khan semble donc remettre en question l'identité du chorégraphique propre au kathak, testant les limites de ce qu'est une danse indienne. Comment peut-on s'éloigner de ce monde gestuel et esthétique sans cesser de produire de la danse indienne? Jusqu'où reste-t-elle encore reconnaissable par le public? Le trouble introduit dans l'identification et dans la reconnaissance des formes traditionnelles apparaît comme la conséquence la plus évidente de son choix.

Bien évidemment, d'autres artistes de la diaspora indienne nous pourrions citer à titre d'exemple Padmini Chattur ou Shantala Shivalingappa - ont recours à ce type de dispositif. Le choix d'interroger Akram Khan répond ici à une motivation esthétique essentielle: l'organisation sensible et perceptive propre à sa corporéité est d'une lisibilité telle que l'analyse s'en trouve facilitée. 


\section{Du Désir de Reconnaître au Défi de Voir}

Dans les pages précédentes, nous avons vu qu'un bon nombre de spectacles hybrides restent affectés par la nécessité de montrer et de désigner l'appartenance à un univers culturel bien défini. L'artiste reste partagé entre l'envie d'oublier toute revendication identitaire et en même temps de la rendre manifeste. Comme le dit Homi Bhabha: "[...] presque le même, mais pas tout à fait" (2007, p. 153). Les Indiens parlent - et il faudrait ajouter dansent - comme les Blancs mais ils gardent un accent, un dessin gestuel qui encore les caractérise. Un tel désir de distinction de son propre vécu identitaire sous-tend une impossibilité de se défaire du concept de moi en tant qu'Indien, en tant que sujet culturellement et physiquement construit et délimité. Ces processus de création proposent un emploi de la technique qui simplement trouble et altère la réception des signes de reconnaissance. Parfois, ces œuvres décontextualisent la pratique, d'autres fois, s'approprient du vocabulaire de départ sous forme de citation. Dans les deux cas, leurs mouvements se donnent en tant que reprise, répétition et forme de survie consciemment organisée et structurée. La spécificité de ces créations se manifeste dans les contradictions inhérentes au désir de voir et dans la volonté de fixer la différence culturelle dans un objet maitrisable et percevable. Une forme de déstabilisation, de confusion, de brouillage est déjà à l'œuvre dans ces créations, qui restent cependant dépendantes des signes identificatoires du langage indien.

La démarche corporelle d'un artiste comme Akram Khan - et bien d'autres - semble vouloir abandonner toute référence explicite à ce bagage gestuel spécifiquement indien. Refusant les formes de reconnaissance immédiates et directes, il met en scène les principes sensibles et qualitatifs du kathak. Sa danse se construit alors à partir des couches les plus profondes de l'imaginaire kinesthésique. Réexpérimentant autrement la posture et la coordination propres à la danse kathak, Akram Khan laisse surgir et apparaître les résonances internes qui informent ces danses. Le jeu consolatoire de la reconnaissance, qui assurait une plénitude de signification, s'avère d'emblée renié et contredit par la perturbation du regard mise en place. Les créations d'Akram Khan conçoivent les formes à partir d'un niveau de visibilité qu'on aimerait qualifier de non-rétinien, mettant en scène des règles de reconnaissance définitivement renversées. Leur 
conception marque le passage d'un désir de voir et de fixer la différence culturelle dans un objet maîtrisable, à un défi de voir. Cette stratégie récuse l'emploi de la citation, pour adopter d'autres cheminements bien plus complexes et éphémères. Chez Akram Khan, la tradition se donne plutôt sous forme de survivance ${ }^{15}$, qui "[...] persiste à travers ses modifications, et signale une [...] permanence d'habitudes motrices dans un contexte bouleversé" (Launay, 2007, p. 243). Loin d'être un élément bien saisissable et manifeste, la survivance est ici de l'ordre de l'imperfection, de ce qui involontairement entrave ou met en crise le bon fonctionnement du dispositif. C'est pour cette raison que la possibilité de la pointer du doigt reste faible et le critère de visibilité rétinienne n'apparaît plus approprié ni applicable.

Ce type de processus de création qui laissent interagir des expériences kinesthésiques distinctes, s'avère pour nous d'un intérêt remarquable pour le dispositif de création qu'il met en place. D'un point de vue esthétique, ces pièces s'éloignent considérablement des créations qui travaillent à partir des formes. Résultant d'un état et d'un travail sensibles et proprioceptifs, ces créations se rapprochent des phénomènes émergents décrits par Francisco Varela. Le processus strictement relationnel qui détermine l'émergence de la forme, pose le spectateur dans une posture particulière, car il se trouve confronté à la difficulté de saisir la complexité du phénomène. Ce processus de création, qui se constitue à partir d'une réorganisation sensible, donne vie à une esthétique spécifique qu'il vaut la peine de distinguer et de nommer. En référence aux théories de Varela, nous aimerions la désigner sous le terme d'esthétique de résonance.

D'un point de vue politique, ces pièces relèvent d'un acte extrême envers les tensions structurelles de l'art indien, faisant basculer les catégories de reconnaissance imposées par la tradition. Ce type de créations présente en effet un processus de composition qui remet littéralement en cause la question du vocabulaire, avec de fortes répercussions sur la façon de penser et de percevoir les danses de l'Inde. Ce type de recherche représente finalement un moyen de s'extraire de l'autorité technique et religieuse imposée par la culture indienne, affirmant la primauté du sujet dansant à l'égard de l'ordre établi. 


\section{Notes}

${ }^{1}$ Le terme hybride indique le "[...] croisement naturel ou artificiel de deux individus d'espèces, de races ou de variétés différentes" (Tresor de la Langue Française, 2014, n.p.). Dès sa conception, ce terme a été investi, d'un côté par l'idée de race et de genèse inhumaine, de l'autre par l'idée d'intervention volontaire sur la nature des choses. L'exemple le mieux connu d'hybride est sûrement le mulet, engendré par l'accouplement d'un âne et d'une jument.

${ }^{2}$ Michel Bernard est le fondateur du département danse de l'Université de Paris 8 Saint-Denis. Prolongeant les perspectives phénoménologiques, son approche s'attache à l'exploration de l'acte de danser, via l'étude des différentes facettes de la corporéité, des processus de création chorégraphique et du phénomène de la réception.

${ }^{3}$ L'expression expérience perceptive fait référence à l'expérience de soi (proprioception ou intéroception) combinée à l'expérience de l'environnement (extéroception).

${ }^{4}$ Précisons à cet égard, que les travaux d'Avanthi Meduri ont contribué à déconstruire l'historiographie nationaliste indienne des années 1940/1950, qui organise son discours autour d'une idée de pureté de la pratique. Avanthi Meduri démontre que des formes d'hybridité étaient déjà à l'œuvre dans la pratique des devadasis: la confrontation avec l'empire colonial anglais, qui, vers la moitié du XVIIIe siècle, envahit les cours de l'Inde, lui permet d'envisager ces danseuses comme des femmes cosmopolites et internationales et leur danse comme une pratique hybride. Ses travaux ont également démontré que le terme sanskrit Bharata Natyam, créé au début du $\mathrm{XX}^{\mathrm{e}}$ siècle, devient au cours des années trente un terme parapluie qui rassemble une pluralité d'expressions corporelles, induisant un égarement linguistique important. Le terme identité locale, utilisé dans son article, fait évidemment référence à cette pluralité de cultures qui coexistent à l'intérieur du SadirBharata Natyam, et plus généralement à l'intérieur de la culture indienne.

${ }^{5}$ La publication de Pulse date de 2000, mais il faut rappeler que cette revue remplace la première publication en danse en UK, the newsletter ADITI, qui date de 1993.

6 "PULSE, which is primarily funded by Arts Council England, serves to highlight, that Britain is a happening place for South Asian dance and sets international trends in performance arts". Disponible sur: <http://www.kadam.org.uk/pulse.php>. Consulté le: 06 jui. 2010.

${ }^{7}$ Rappelons que la danse anglo-saxonne des années 1980 établit un lien très étroit avec les études postcoloniales. En tant que terrain théorique vif et fertile au sujet des questions identitaires, les réflexions propres à ce champ d'étude s'affirmeront comme référence privilégiée à l'apparition d'un discours théorique sur la danse de la diaspora.

${ }^{8}$ Philosophe indien naturalisé américain, Homi Bhabha a été fortement influencé par le poststructuralisme occidental et surtout par les écrits de Jacques Derrida, Jacques Lacan et Michel Foucault. Le discours de Homi Bhabha vise la déconstruction des confins entre soi et les autres, voire entre les sujets coloniaux et les sujets colonisés. Se soustrayant à une vision dichotomique simple et rassurante, son intérêt se focalise sur l'espace qui relie les cultures de référence, qu'il nomme tiers espace. Lieu de production de différences internes, de déplacements de sens inattendus et fortuits, cet inter-est, cet espace entre est le lieu qui 
permet d'"[...] éluder la politique de la polarité et enfin [d'] émerger comme les autres de nous-mêmes" (Bhabha, 2007, p. 19).

${ }^{9}$ À ce propos, nous renvoyons le lecteur au livre de De Oliveira, Oxley et Petry qui parle de l'installation comme d'une "[...] discipline hybride" (1997, p. 7).

${ }^{10}$ Les théories de Humberto Maturana et de Francisco Varela s'inscrivent dans un cadre intellectuel plus vaste, qui se soucie de donner des réponses aux phénomènes d'élaboration et de transformation de la réalité existante. Plusieurs personnalités du monde de la science et de la littérature ont élaboré des réflexions qui s'avèrent voisines de leurs spéculations. Sous le nom de théorie de l'information, ces conceptions se développent autour des années 1940-1950 grâce à Shannon et Weaver. Il faut spécifier que la théorie de l'information identifie comme bruit toute perturbation interne ou externe à n'importe quel système. Dans un système simple, un bruit représente simplement un problème au bon fonctionnement de l'organisme. Il empêche la conservation et la rénovation de ses fonctions vitales. Mais dans les systèmes complexes un bruit constitue une impulsion au développement du système, induisant la formation d'une structure plus complexe. Le système complexe est capable de transformer une perturbation initiale - intentionnelle ou involontaire - dans une signification originale et inattendue. De tels organismes prouvent donc une capacité remarquable à l'auto organisation et à la gestion interne des obstacles qui peuvent miner leur fonctionnement.

11 "[...] exists to create and present work that maintains the authenticity of Indian classical dance and music whilst presenting these forms in innovative ways and within contemporary contexts". Citation disponible sur: <http://www.srishti.co.uk>. Consulté le: 05 jui. 2013.

${ }^{12}$ Akram Khan s'inscrit à la De Montford University à Lester en Danse Contemporaine, université dans laquelle il reste pendant deux ans. Puis il entre à la Northern School of Contemporary Dance à Leeds, où il obtient son diplôme avec un first class honours.

${ }^{13}$ Akram Khan a été formé à la danse kathak par le maître Sri Pratap Pawar.

14 "I realised that my body was making its own decision, subconsciously, and a language was emerging that has a balance of both worlds", Massive Kathak, The Sunday Times, April 6, 2003.

${ }^{15}$ La notion de survivance (Nachleben) est le motif central de l'approche anthropologique de l'art occidental élaborée par Aby Warbours. Cette notion a été introduite dans les études en danse par Isabelle Launay, qui a repensé l'histoire de la danse à partir d'un temps nonlinéaire et anachronique où le geste persiste au-delà des circonstances qui l'ont produit, désorientant le temps. Elle parle à ce propos d'une "[...] poétique des gestes revenants" (Launay, 2007, p. 245).

\section{Références}

BATT, Noëlle; ABRIOUX, Yves; CORDESSE, Gérard. Entretien avec Francisco Varela. Théorie, Littérature, Enseignement (TLE), Paris, Presses Universitaires de Vincennes, n. 8, p. 165-175, automne, 1990. 
BERNARD, Michel. De la Création Chorégraphique. Pantin: CND, 2001.

BHABHA, Homi. Les Lieux de la Culture: une théorie postcoloniale. Paris: Payot, 2007.

DE OLIVEIRA, Nicolas; OXLEY, Nicolas; PETRY, Michael. Installations, l'Art en Situation. Paris: Thames \& Hudson, 1997.

FRIMAT, François. Qu'est-ce que la Danse Contemporaine? Paris: PUF, 2010.

JORDAN, Stéphanie. Cultural Crossing: containing the crisis... and the Indian in our midst. In: PONTBRIAND, Chantal (Org.). Danse: langage propre et métissage culturel. Canada: Parachute, 2001. P. 111-120.

KHAN, Naseem; SUNDARAM, Chitra; WOLLASTON, Ginnie; RAY, Piali. Moving Margins: South Asian dance in the UK. Narthaki, [s.l.], fév. 2001. Disponible sur: <http:// www.narthaki.com/info/articles/article13.html>. Consulté le: 08 mars 2014.

KHAN, Akram. Massive Kathak. The Sunday Times, London, Times Newspapers Ltd., s.p., 06 avr. 2003.

LAPLANTINE, François; NOUSS, Alexis. Métissages. De Archimboldo à Zombi. Montréal: Pauvert, 2001.

LATOUR, Bruno. Nous n’avons jamais été Modernes. Paris: La Découverte, 1997.

LAUNAY, Isabelle. Les Danses d'après, pour une Poétique de la Mémoire en Danse. Dossier d'HDR, Saint-Denis, Département Danse, Université de Paris 8 - Saint Denis, déc. 2007.

LOPEZ Y ROJO, Alessandra. Dance - Space - Architecture. Pulse, Angleterre, n.p., été, 2006. Disponible sur: <http://humanitieslab.stanford.edu/49/75>. Consulté le: 10 jui. 2013.

MEDURI, Avanthi. The Transfiguration of Indian/Asian Dance in the United Kingdom: contemporary Bharatanatyam in global contexts. Asian Theatre Journal, États-Unis, University of Hawaii Press, v. 25, n. 2, p. 298-328, 2008.

MOHAIEMEN, Naeem. Akram Khan: explosion in contemporary dance. The Daily Star, Bangladesh, 27 avr. 2003. Disponible sur: <http://www.akramkhancompany.net/ html/akram_article.php?id=55>. Consulté le: 08 avr. 2013.

MOLINET, Emmanuel. L'Hybridation: un processus décisif dans le champ des arts plastiques. Le Portique, France, Association Le Jardin, n. 2, p. 1-16, 22 déc. 2006. Disponible sur: <http://leportique.revues.org/851>. Consulté le: 02 avr. 2014.

PICCIRILLO, Annalisa. Hybrid Bodies in Transit: the 'third language' of contemporary kathak. Anglistica, Napoli, Università degli Studi di Napoli "L'Orientale", v. 12. n. 2, p. 27-41, 2008. Disponible sur: <http://www.anglistica.unior.it/sites/anglistica/files/04\%20 Piccirillo.pdf>. Consulté le: 20 mars 2014.

ROY, Sanjoi. Growths and Outgrowths. In: KOTHARI, Sunil. New Direction in Indian Dance. Mumbai: Marg Publication, 2003. P. 156-167.

ROLNIK, Suely. L'Hybride de Lygia Clark. Traduction: Alain Mouzat. Caosmose, [s.l.], 15 fév. 2004. Disponible sur: <http://caosmose.net/suelyrolnik/pdf/hybride.pdf >. Consulté le: 20 juin 2013. 
TRESOR DE LA LANGUE FRANÇAISE. Disponible sur: <http://atilf.atilf.fr/dendien/ scripts/tlfiv5/advanced.exe?8;s=551496660>. Consulté le: 15 mai 2014.

VARELA, Francisco. La Coscienza nelle Neuroscienze. Conversation avec Sergio Benvenuto, faite par RAI Educational. Enciclopedia Multimediale delle Scienze Filosofiche, Paris, Laboratoire Lena, Hôpital de la Pitié-Salpêtrière, 29 jan. 2001. Disponible sur: <http:// www.psychomedia.it/pm/science/psybyo/varela.htm>. Consulté le: 20 juin 2013.

VARELA, Francisco. Conférence. [S.1.], TV Atina, sep. 2008. Disponible sur: <http:// www.youtube.com/watch?v=bujfwhEsF04\&feature=related>. Consulté le: 25 juin 2013.

Federica Fratagnoli est docteur en Esthétique, Sciences et Technologies des Arts, spécialité Danse, à l'Université de Paris 8 - Saint Denis. Actuellement elle est Maître de conférences en danse à l'Université de Nice Sophia Antipolis, membre du Centre Transdisciplinaire d'Épistémologie de la Littérature et des Arts Vivants (CTEL) et membre associé du Laboratoire d'analyse des Discours et Pratiques en Danse de l'Université Paris 8 Saint-Denis.

E-mail: federicafratagnoli@hotmail.com

Texte original et inédit en français, révisé par Annelyse Gayraud. Une traduction en portugais est publiée dans ce même numéro de la revue. 